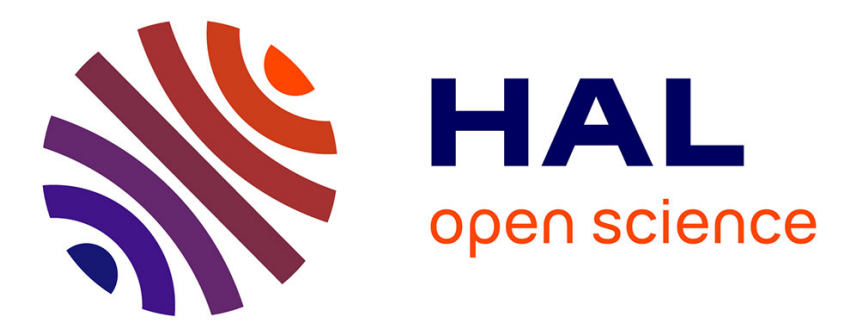

\title{
Hydrodynamics and bubble size in bubble columns: effects of contaminants and spargers
}

L. Gemello, C. Plais, F. Augier, A. Cloupet, D.L. L Marchisio

\section{To cite this version:}

L. Gemello, C. Plais, F. Augier, A. Cloupet, D.L. L Marchisio. Hydrodynamics and bubble size in bubble columns: effects of contaminants and spargers. Chemical Engineering Science, 2018, 184, pp.93 - 102. 10.1016/j.ces.2018.03.043 . hal-01899599

\section{HAL Id: hal-01899599 \\ https://hal-ifp.archives-ouvertes.fr/hal-01899599}

Submitted on 19 Oct 2018

HAL is a multi-disciplinary open access archive for the deposit and dissemination of scientific research documents, whether they are published or not. The documents may come from teaching and research institutions in France or abroad, or from public or private research centers.
L'archive ouverte pluridisciplinaire HAL, est destinée au dépôt et à la diffusion de documents scientifiques de niveau recherche, publiés ou non, émanant des établissements d'enseignement et de recherche français ou étrangers, des laboratoires publics ou privés. 


\title{
Hydrodynamics and bubble size in bubble columns: effects of contaminants and spargers.
}

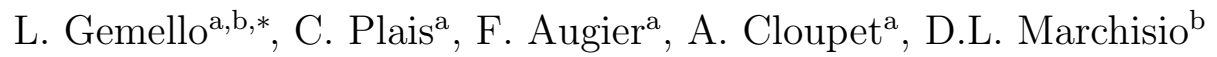 \\ ${ }^{a}$ IFP Energies nouvelles, Rond-point de l'échangeur de Solaize, 69360 Solaize (69), \\ France \\ ${ }^{b}$ Dipartimento di Scienza Applicata e Tecnologia, Istituto di Ingegneria Chimica, \\ Politecnico di Torino, Corso Duca degli Abruzzi 24, 10129 Torino (TO), Italy
}

\begin{abstract}
The simulation of bubble columns operating under the heterogeneous regime is an ambitious challenge, due to the difficulty of predicting accurately hydrodynamics and bubble size distributions, that requires experimental data for model validation. Gas fraction distributions, liquid and gas velocity profiles and bubble size distributions across bubble columns are deeply interconnected in these systems and only a comprehensive study allows the links between them to be understood. This work reports experimental data obtained by measuring bubble sizes with an innovative technique based on the cross correlation between two optical probes. Particular attention is given to the role of additives and impurities with a view to suppressing bubble coalescence. Initially experiments are carried out with demineralized water; subsequently they are repeated with tap water and adding small quantities of ethanol. Results show that contaminants and alcohol addition suppress bub-
\end{abstract}

\footnotetext{
${ }^{*}$ Corresponding author

Email addresses: luca.gemello@polito.it (L. Gemello), cecile.plais@ifpen.fr (C. Plais), frederic.augier@ifpen.fr (F. Augier), ann.forret@ifpen.fr (A. Cloupet), daniele.marchisio@polito.it (D.L. Marchisio)
} 
ble coalescence and induces a decrease of mean bubble sizes. Furthermore, alcohol addition delays the transition from homogeneous to heterogeneous regimes and increases the gas hold-up under the heterogeneous regime. Gas distribution is studied through two different perforated spargers. Changing the sparger it is possible to modify the bubble size in the lowest part of the column significantly. A perforated sparger with big holes causes the formation of big bubbles close to the holes of the sparger and promotes bubble breakage in the lower part of the column. By combining ethanol addition and sparger modification, bubble coalescence and bubble breakage can be decoupled in a controlled manner and interesting conclusions concerning these processes can be drawn. Beyond the novelty of bubble size measurements at high gas fraction, the experimental data collected are very useful to validate and develop computational fluid dynamics simulations coupled with population balance models suitable for heterogeneous bubbly flows.

Keywords: Bubble columns, Heterogeneous regime, Contamination, Alcohol, Sparger

\section{Introduction}

Bubble columns are often used as gas-liquid reactors, especially for reactions with slow kinetics such as oxidation, chlorination, alkylation, hydroformylation, hydrogenation, Fischer-Tropsch synthesis, coal liquefaction, desulfurization and fermentation (Chaumat et al., 2007; Ranade, 2002). These reactors may also be used for waste water treatment, cell cultures, production of organic acids or yeasts (Tisnadjaja et al., 1996). Bubble column reactors have low operation costs, low maintenance and good mass and heat 
transfer rates, but their hydrodynamics is complex and strongly depends on geometry, fluid flow rates and potential presence of internals.

For decades, scale-up of bubble columns was based on the use of macroscopic correlations to describe hydrodynamics and transport (Deckwer, 1992; Xiao et al., 2017). Nowadays, Computational Fluid Dynamics (CFD) appears as a promising tool to predict global and local properties of interest in bubbly flows (Jakobsen et al., 2005), overcoming the constraints of the traditional scale-up approach, such as the systematic use of expensive experimental setups of increasing sizes. Although numerical tools are very promising, they still need development to be fully useful in some complex flow configurations, as it is the case for industrial bubbly flows at high gas fraction. The bubble size distribution (BSD) is a key parameter for bubble column reactor performance, but until recently it was still a challenge to measure properly BSD beyond a few percentage of gas hold-up (Xue, 2004; Chaumat et al., 2007; Maximiano Raimundo, 2015; McClure et al., 2017). The prediction of BSD by CFD models is thus an exciting challenge as it would help make CFD completely predictive. Only a very few papers deal with BSD measurements in the heterogeneous regime (Xue, 2004; Chaumat et al., 2007; McClure et al., 2015, 2016, 2017). They are based on the calculation of chord distributions by multi-point needle probes. This technique has been validated in case of bubbles having almost vertical trajectories. In case of heterogeneous regime, bubbles do not have vertical trajectories, especially when the distance from the column center increases, causing low accuracy (McClure et al., 2013; Xue, 2004). Despite this limitation, multi-point probes have the advantage of accessing both the bubble chord distribution and the bubble velocity. 
The BSD can be numerically predicted when CFD is coupled with a Population Balance model (PBM), solved for example with a Quadraturebased Moments Method (QBMM) approach (Marchisio and Fox, 2013) to limit the number of equations to solve. Ideally bubble coalescence (Liao and Lucas, 2010) and bubble breakage (Liao and Lucas, 2009) ought to be decoupled in order to study them separately.

In this context, experimental data collected in the present work have a particular interest for the development and the validation of PBM in the heterogeneous regime. In fact, most of the experimental published works are concentrated on the homogeneous bubbly flow regime (Krishna, 2000) at low superficial gas velocities. In the present work several properties are measured under the heterogeneous regime: local and global gas fractions, liquid velocity and bubble size.

To overcome the limitations of chord-based measurement techniques, a cross-correlation (CC) technique has been recently developed to measure the bubble size independently of the bubble trajectory. It is thus well adapted to heterogeneous regimes (Maximiano Raimundo, 2015; Maximiano Raimundo et al., 2016). However CC techniques do not provide chord distributions, but a mean diameter, that is measured with confidence at any radial position. This technique is therefore complementary to the existing ones, providing a reliable average measurement for every position of the column.

An important issue concerning bubbly flows is the impact of additives and impurities. Industrial applications never involve pure fluids, as generally liquids of complex compositions are employed. A few experimental well-documented works provide data on bubble properties in the presence of 
additives (McClure et al., 2015). Alcohol addition delays the transition of bubbling regimes to higher gas velocity, due to the decrease of coalescence (Keitel and Onken, 1982; Guo et al., 2017) and the increase of bubble rigidity (Dargar and Macchi, 2006). This effect rises gradually with alcohol molecular weight (Jamialahmadi and Miiller-Steinhagen, 1992). The decrease of surface tension in the presence of alcohol is negligible or at least not sufficient to explain the magnitude of this phenomenon; this difference is due to the reduction of the bubble-rise velocity, as suggested by several authors (Lindland and Terjesen, 1965; Raymond and Zieminski, 1971; Clift et al., 1978; Kelkar et al., 1983). Another point of industrial interest concerns the effect of the sparger on the bubble size, which has been reported in some articles (Chaumat et al., 2007; McClure et al., 2016). The choice of the sparger appears as a way to modify the BSD at the entrance of the column. Polli et al. (2002) stated that the effect of the sparger persists for a height of approximately one column diameter.

The goal of this work is to produce data that will allow the validation of bubble coalescence and breakage models, by realizing experiments in which coalescence and breakage change depending on the operating conditions. Demineralized water, tap water and demineralized water with different ethanol concentrations are employed to suppress bubble coalescence. Experiments are performed using two different spargers, generating very different initial bubble sizes and simulating in some cases strong bubble breakage. By considering simultaneously additives and spargers effects, breakage and coalescence phenomena can be decoupled. Besides the mean diameter profiles, gas fraction and liquid velocity distributions are measured, providing a very 
useful and accurate set of experimental data. The cross-correlation technique was first presented by Maximiano Raimundo (2015) and it was used only with tap water and air. In this paper, this technique is employed under the presence of additives and impurities. It is the first time in which this technique is used for evaluating contamination, ethanol and sparger effects at high gas fraction for every radial and axial position.

Eventually some perspectives concerning computational fluid dynamics simulations and population balance modelling are also discussed. The main novelties of this work are linked to the role of additives and impurities for suppressing bubble coalescence and the effects of the sparger with a view to decoupling coalescence and breakage phenomena for population balance modelling.

\section{Experimental setup}

Experiments are conducted in a $0.4 \mathrm{~m}$ diameter cylindrical bubble column reactor (Fig. 1a) for a wide range of superficial gas velocities, from $3 \mathrm{~cm} / \mathrm{s}$ to $35 \mathrm{~cm} / \mathrm{s}$. All the experiments are carried out at atmospheric pressure. The initial height of liquid into the column (without gas) is equal to 4 diameters. If this ratio is above a critical value, as in this case, the gas fraction does not depend on the initial height (Sasaki et al., 2017). The total height of the column is higher than 8 diameters, in order to work with very high gas fraction. There is no net flow rate of liquid during experiments.

The gas is injected through a perforated sparger from the bottom of the column. The sparger has 92 holes of $2 \mathrm{~mm}$ of diameter (Fig. 1b), ensuring a homogeneous distribution of the gas. Subsequently, another perforated 


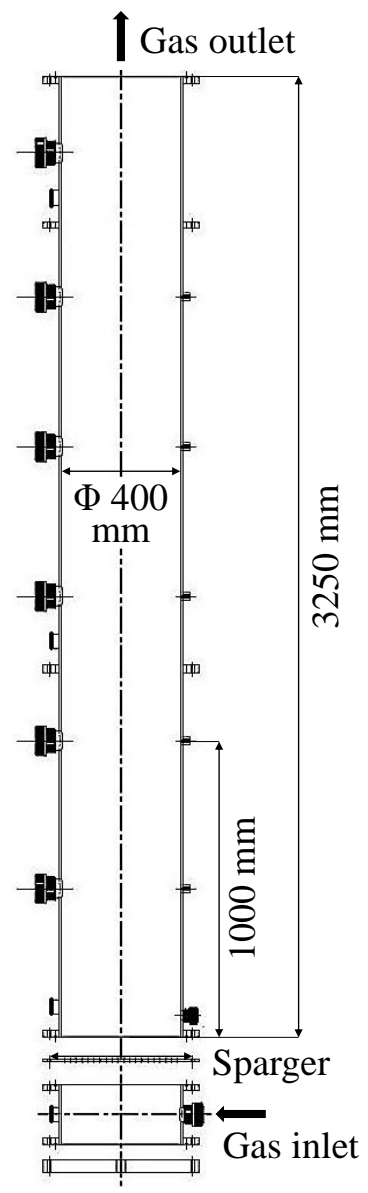

(a) Axial section of the bubble column
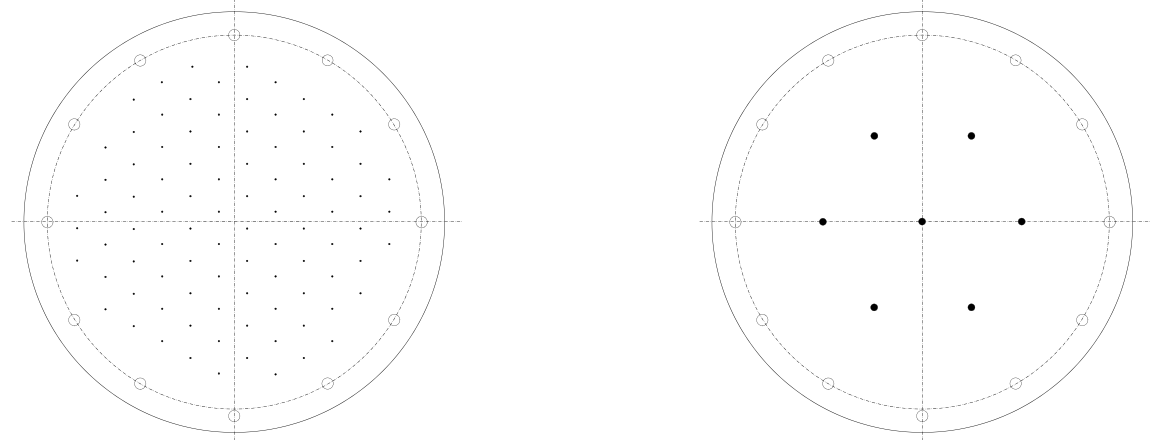

(b) Perforated sparger with 92 holes of $2 \mathrm{~mm}$

(c) Perforated sparger with 7 holes of $9 \mathrm{~mm}$

Figure 1: Bubble column and spargers. 
sparger is used (7 holes of $9 \mathrm{~mm}$ ) (Fig. 1c): this allows the study of sparger effects on hydrodynamics and bubble size. As shown in Figure 1c, one hole is in the center of the sparger, while the other 6 holes create a hexagon for a dimensionless radial position equal to 0.65 .

Different qualities of water are used in this work: demineralized water (that can be considered slightly contaminated water or pure water), tap water (fully contaminated water) and small percentages of ethanol $(0.01 \%$ and $0.05 \%$ in volume) in demineralized water.

The global gas hold-up, also called gas hold-up or global void fraction, is calculated by using the difference of liquid height before and after gas injection. This method is not very accurate due to the deformation of the interface at the top of the column: the characteristic error associated with these measurements is of about 10\% (Maximiano Raimundo, 2015).

A common method to obtain the local gas fraction is to use an optical probe, that detects the presence of gas or liquid. The local gas hold-up is calculated as the percentage of time in which the gas is detected at the tip of the probe (Maximiano Raimundo, 2015). Forret (2003) measured liquid velocity in bubble columns of different diameters in the heterogeneous regime by using a modified Pitot tube, called a Pavlov tube. The differential pressure between two opposite holes allows to obtain the liquid velocity. Due to the presence of two phases, Vial et al. (2003) suggest adding a calibration parameter and to consider a correction term that takes into account the gas fraction. The error associated to these measurements is of about $10-20 \%$, depending on the gas fraction.

The mean bubble size is obtained from the spatial cross-correlation (CC) 
of the signals of two optical probes. The cross-correlation quantifies the similarity of two signals, analyzing them simultaneously. Its value is maximal (and equal to 1) if the two probes are at the same point, since the signals are equal. The cross-correlation decreases with the distance between the probes until reaching an asymptotic value, which depends on the gas fraction into the column. The time-averaged horizontal bubble diameter can be obtained from the $\mathrm{CC}$ calculation, the distance between probes and the eccentricity of the bubbles. The two probes are located at the same horizontal level and separated by a distance of $1 \mathrm{~mm}$. This method is suitable for heterogeneous regime and high gas fraction (see Maximiano Raimundo (2015) and Maximiano Raimundo et al. (2016) for details), as it has been successfully validated in comparison with endoscopic measurements. The error does not exceed $10 \%$ for each operating condition. Bubbles in the case of the heterogeneous regime are mostly oblates. The cross-correlation technique measures the horizontal diameter, using the average of Sauter between the different bubbles. A constant eccentricity of 0.7 is considered: this value is obtained from the experiments of Maximiano Raimundo (2015) in the same range of measured bubble size. By knowing the shape of the bubbles, it is possible to calculate the time-averaged Sauter diameter, also called mean Sauter diameter, starting by the time-averaged horizontal bubble diameter. The considered eccentricity is rather in accordance with the value predicted with the correlation of Wellek et al. (1966) for liquid drops, that depends on the Eötvös number and subsequently on the surface tension. 


\section{Results and discussion}

\subsection{Hydrodynamics: liquid velocity and gas fraction}

Initially, the sparger with holes of $2 \mathrm{~mm}$ is used. The global gas hold-up changes with the superficial gas velocity and its change allows to distinguish the homogeneous and the heterogeneous regimes. The gas hold-up is also affected by the bubble size: big bubbles rise more rapidly than the smaller ones.

Using demineralized water, the regime is homogeneous until a superficial gas velocity of $5 \mathrm{~cm} / \mathrm{s}$, as shown in Fig. 2, where the measured gas hold-up is reported versus the superficial gas velocities for demineralized water, tap water and demineralized water with different concentration of ethanol. Up to $5 \mathrm{~cm} / \mathrm{s}$, the gas hold-up increases quickly with a linear dependence on the superficial gas velocity. After this critical value, the transitional regime takes place. During the transitional regime, the gas hold-up dependence versus the superficial gas velocity is weaker. The hold-up curve has a monotonic increasing trend, without any local maximum in the transitional regime. The heterogeneous regime begins at a superficial gas velocity of about $9 \mathrm{~cm} / \mathrm{s}$, when the gas hold-up starts to increase with a quite linear dependence.

In the case of contaminated water, the gas hold-up curve has some discrepancies corresponding to the transitional regime, as reported in Fig. 2. The transitional regime behavior depends on the water supply quality during the column filling and therefore results are not fully reproducible. It is interesting to observe however that far away from the transitional regime, the trends for demineralized water and tap water are very similar. The addition of small quantities of ethanol in water, changes radically the hydrodynam- 


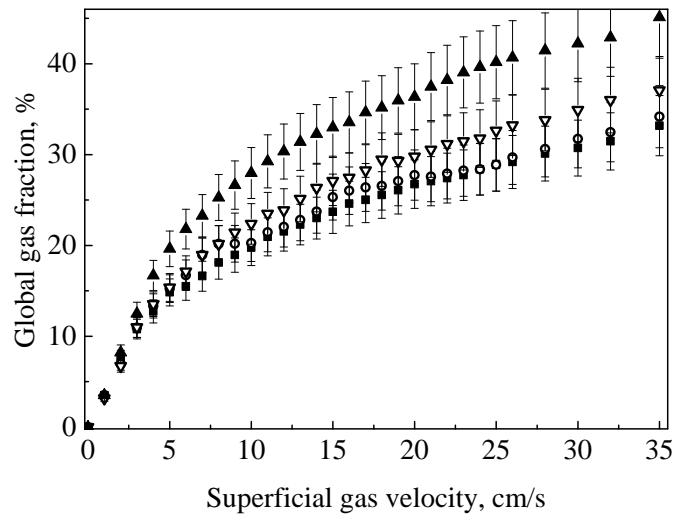

Figure 2: Gas hold-up versus superficial gas velocity for demineralized water (घ), tap water $(\circ)$, demineralized water with ethanol $0.01 \%(\nabla)$ and demineralized water with ethanol $0.05 \%(\mathbf{\Lambda})$.

ics especially in the heterogeneous regime. The trend is similar under the homogeneous regime, but the transition is delayed, as reported in Fig. 2. These discrepancies are more obvious when the concentration of ethanol is higher. Under the heterogeneous regime, the difference of gas hold-up between ethanol solutions (at a fixed concentration) and demineralized water is almost constant regarding the variation of the superficial gas velocity.

The liquid velocity profile is influenced by global hydrodynamics and it is linked to bubbles recirculation (Krishna et al., 2000b). The radial profile has a quadratic shape under the heterogeneous regime, with a strong liquid recirculation, that rises in the center and goes down next to the wall (Fig. 3). The mean axial liquid velocity profile and the recirculation does not seem to be influenced by the presence of contaminants into water. The velocity profile depends on the vertical position close to the sparger, while the dependence is negligible in case of $\mathrm{H} / \mathrm{D}>1$. 


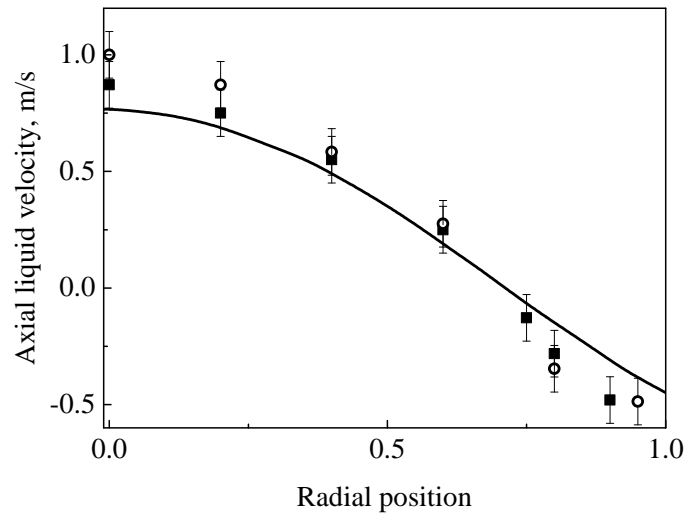

Figure 3: Axial liquid velocity radial profile for a superficial gas velocity of $16 \mathrm{~cm} / \mathrm{s}$ at $\mathrm{H} / \mathrm{D}=2.5$ for demineralized water $(\boldsymbol{\square})$ and tap water $(\circ)$; radial profiles are compared with the correlation of Miyauchi and Shyu (1970) coupled with the correlation of Forret (2003) $(-)$.

The experimental data of the liquid velocity profile are compared with the correlation of Forret (2003):

$$
u_{l}(r)=\frac{u_{l}(0)}{a-c}\left[a \exp \left(-b r^{2}\right)-c\right]
$$

with $\mathrm{a}=2.976, \mathrm{~b}=0.943$ and $\mathrm{c}=1.848$, empirical parameters determined at ambient conditions in a wide range of validity under the heterogeneous regime. They were established by Forret (2003) thanks to several measurements of the axial liquid velocity with a Pavlov tube in bubble columns with a diameter between $0.15 \mathrm{~mm}$ and $1 \mathrm{~m}$.

This equation is empirical, but it allows to define correctly the shape of the radial velocity profile if the liquid velocity in the center of the column is known. The upward flow rate is equal to the downward one, in accordance with experimental data. This correlation is also suitable for contaminated systems and in presence of additives. 
The liquid velocity in the center of the column is obtained with the correlation proposed by Miyauchi and Shyu (1970):

$$
u_{l}(0)=2.47 v_{s g}^{0.5} D^{0.28},
$$

where $v_{s g}$ is the superficial gas velocity.

This correlation is empirical, but it has a wide range of validity in the so called stabilized region $(H / D>1)$. This equation can be used for obtaining a first approximation of the axial liquid velocity in the center for many operating conditions under the heterogeneous regime. This correlation can be also used for scale-up purposes. Unfortunately, this correlation cannot be used for contaminated systems and in presence of additives. This correlation slightly underestimates the liquid velocity in the center of the column also without additives. CFD simulations could overcome the limits of this correlation. This set of experimental data will allow to carry out CFD simulations for testing models that could consider the presence of additives and that could be used in a wider range of operating conditions for each position of the bubble column.

The local gas fraction profile depends strongly on radial position, axial position and superficial gas velocity. Increasing the superficial gas velocity, the local gas fraction become higher in each position of the bubble column. The effect is important in the center of the column, where the largest bubbles rise to the top. Near the wall small bubbles are carried down by the liquid recirculation. In the homogeneous regime $(3 \mathrm{~cm} / \mathrm{s})$ the radial profile is not very pronounced, while in the heterogeneous regime and in the transitional regime, the radial profile has a stronger concavity (Fig. 4). The radial profiles of gas faction may depend on interfacial momentum transfer between gas and 


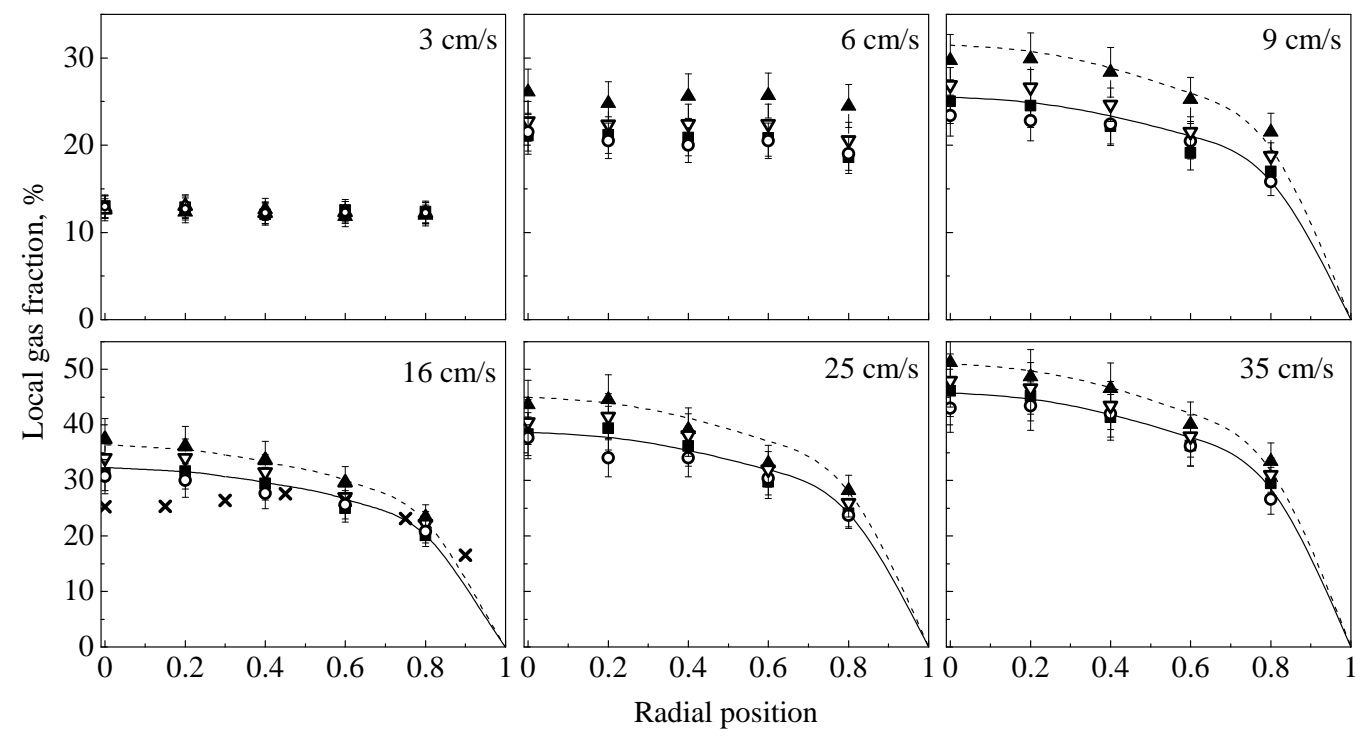

Figure 4: Radial gas fraction profiles at $\mathrm{H} / \mathrm{D}=2.5$ with demineralized water ( $\mathbf{\square})$, tap water $(\circ)$, ethanol $0.01 \%(\nabla)$ and ethanol $0.05 \%(\mathbf{\Lambda})$ at different superficial gas velocities. Comparison with the experimental data of McClure et al. (2016) $(\times)$ and with Schweitzer (2001) correlation profiles for water (-) and ethanol 0.05\% (- - -).

liquid.

The influence of the tap water contamination on the gas fraction is low for the homogeneous regime and the heterogeneous regime (Fig. 2). Adding ethanol, with a superficial gas velocity of $6 \mathrm{~cm} / \mathrm{s}$, the radial profile is approximately flat: the transition is delayed. Under the homogeneous regime, the local gas fraction is almost not influenced by the ethanol addition. Under the heterogeneous regime, the local gas fraction is higher under the presence of small quantities of ethanol (Fig. 4).

The axial gas fraction profile in the center of the column increases along the axial position, as shown in Fig. 5. It means that more gas is trapped in the highest part of the column. This effect is present under both the 
homogeneous and the heterogeneous regimes.

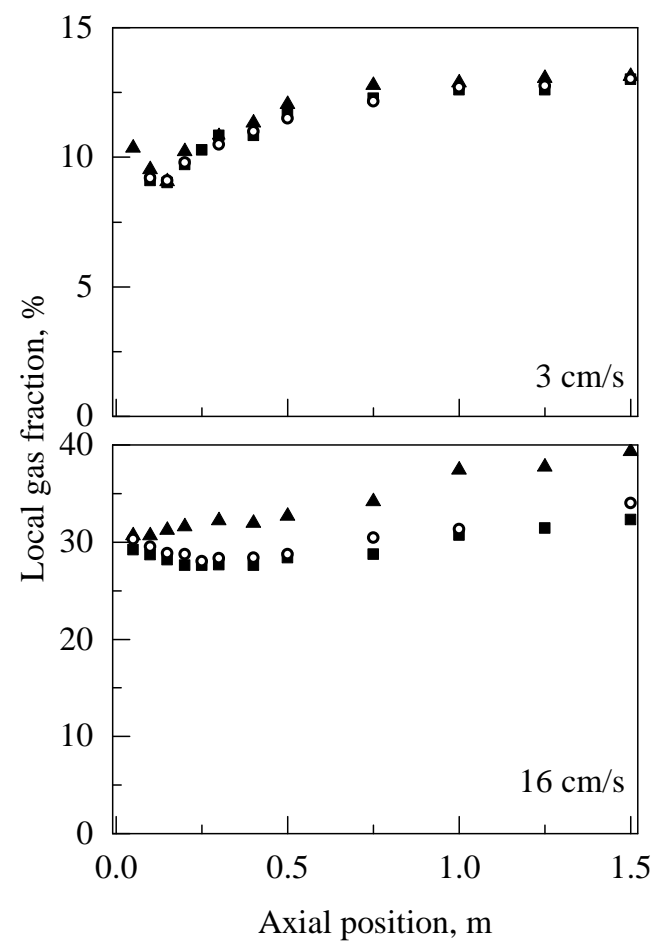

Figure 5: Axial gas fraction profile in the center of the column with demineralized water (๘), tap water (o) and ethanol $0.05 \%$ ( $\mathbf{\Delta})$ with two different superficial gas velocities: 3 $\mathrm{cm} / \mathrm{s}$ (top) and $16 \mathrm{~cm} / \mathrm{s}$ (bottom).

For every position, in the case of heterogeneous regime, the local gas fraction increases adding a small quantity of alcohol: the discrepancy in terms of gas fraction is important both in the case of radial profile (Fig. 4) and along the axial position (Fig. 5). The ethanol effect on the gas fraction is null under the homogeneous regime.

In order to further analyze the local gas fraction measurements, they are initially compared with the correlation of Schweitzer (2001), adapted to the prediction of gas fraction profiles under the heterogeneous regime. It does 
not consider the axial effect and it is expressed as follows:

$$
\alpha_{g}=\bar{\alpha}_{g}\left[a\left(r^{6}-1\right)+b\left(r^{4}-1\right)+c\left(r^{2}-1\right)\right]
$$

where $r$ is the dimensionless radial position, $\bar{\alpha}_{g}$ is the mean gas fraction and $a=-1.7889, b=1.228$ and $c=-0.939$ are fitting parameters.

This correlation considers that the local gas fraction near the wall is close to zero, as confirmed by the experiments of Forret (2003). The correlation of Schweitzer (2001), calculating the mean gas fraction as the average in the axial position of interest, fits well the experimental profile in the case of heterogeneous regime and demineralized water (Fig. 4). The correlation of Schweitzer (2001) is applied also in the case of tap water and alcohol addition: the mean gas fraction changes, but knowing it, a correct radial profile of the gas fraction is obtained (Fig. 4).

Results are compared qualitatively with experimental data of McClure et al. (2016). They use 'tree' type spargers, located $135 \mathrm{~mm}$ above the bottom. The radial profiles with this type of sparger have an M-shape in the case of symmetric sparger (Fig. 4). A perforated plate injects gas homogeneously, while a 'tree' type sparger does not. The experimental data dissimilarities between this work and McClure et al. (2016) are mainly due to the different type of sparger used. This comparison shows that even under the heterogeneous regime, the sparger type can significantly modify the global hydrodynamics in the column.

\subsection{Bubble size}

The mean Sauter diameter is obtained from the cross-correlation (CC) measurements for different water qualities, in a wide range of superficial gas 
velocities from $3 \mathrm{~cm} / \mathrm{s}$ to $35 \mathrm{~cm} / \mathrm{s}$. Initially the "homogeneous" sparger is used.

The radial profile of the mean Sauter diameter is symmetrical with a maximum in the center, as shown in Fig. 6. In the case of homogeneous regime, the differences between the center of the column and near the wall are small. Increasing the superficial gas velocity, the concavity grows. Under the heterogeneous regime, big bubbles are formed and they rise in the center of the column, while small bubbles come down close to the wall, due to the liquid recirculation. It is possible to observe that the radial profiles of bubble size, liquid velocity and gas fraction are strongly interconnected.

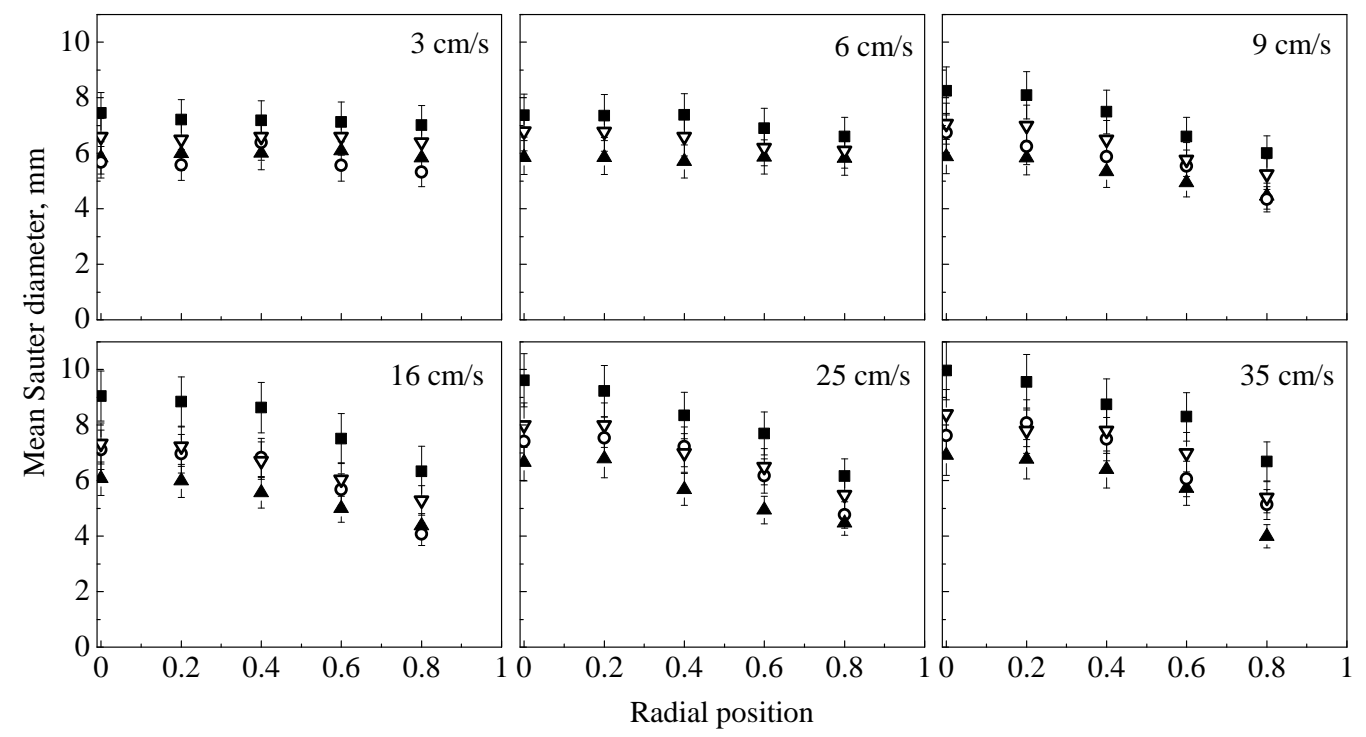

Figure 6: Radial mean Sauter diameter profile at $\mathrm{H} / \mathrm{D}=2.5$ with demineralized water $(\boldsymbol{\square})$, tap water (०), ethanol $0.01 \%(\nabla)$ and ethanol $0.05 \%(\mathbf{\Lambda})$.

The axial evolution of the bubble size at the center of the column is reported in Fig. 7. The mean Sauter diameter decreases slightly for both 
media close to the sparger. Above a certain height, the bubble size grows along the axial position in the case of demineralized water. Close to the bottom, the bubble size profile is not developed and it needs about $50 \mathrm{~cm}$ to reach a stable radial profile; starting from this height, the bubble size in the center of the column grows (Fig. 7). This increase can be due to the decrease of pressure along the axial position. Using the ideal gas law, to obtain a first approximation valuation, it is possible to demonstrate that this effect is less than $5 \%$, while experimentally the increase is more than $20 \%$, implying that other factors are involved. This phenomenon is still difficult to explain but one part of the explanation may be the following: the radial profile is more and more concave along the axial position, causing an increasing of the parabola maximum and the phenomena of bubble coalescence play an important role along the entire column.

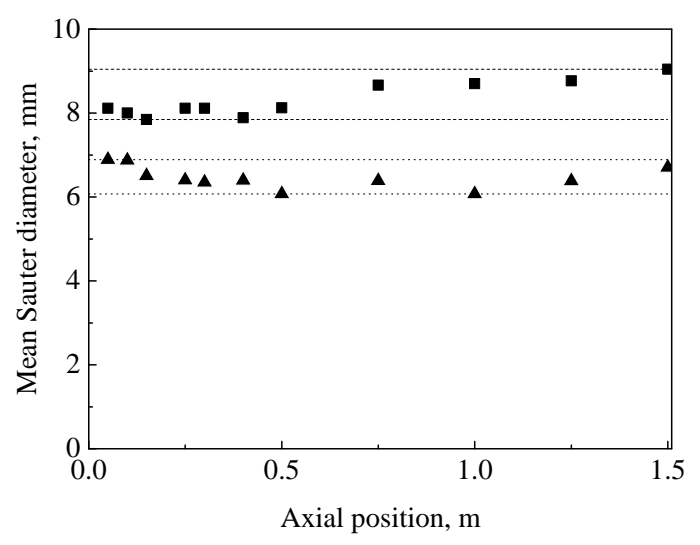

Figure 7: Axial mean Sauter diameter profile in the center of the column for a superficial

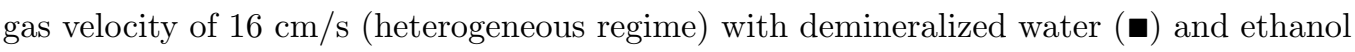
$0.05 \%(\mathbf{\Lambda})$.

Water contamination and presence of additives have an important role on 
the bubble size: for each operating condition, the bubble size in the case of demineralized water is higher than in the case of tap water (Fig. 6). Adding ethanol, the mean Sauter diameter becomes lower in comparison with the case of demineralized water, but it has in any case an upward trend with the superficial gas velocity. The radial profile is almost flat both for $3 \mathrm{~cm} / \mathrm{s}$ and for $6 \mathrm{~cm} / \mathrm{s}$ (Fig. 6), confirming the delay of the transitional regime, as suggested by the local gas fraction profile. The bubble size diminishes with increasing the concentration of ethanol. The experimental results are consistent with the literature values. Ethanol addition and presence of impurities in tap water have similar effects: they decrease coalescence and diminish the mean Sauter diameter. The ethanol effect is consistent with the results of McClure et al. (2015), adding 2-propanol into water.

The surface tension decreases adding ethanol into water. At $17{ }^{\circ} \mathrm{C}$, with a concentration of $0.05 \%$, the surface tension decreases from $73.35 \mathrm{mN} / \mathrm{m}$ to $73.25 \mathrm{mN} / \mathrm{m}$. The difference is negligible and the decrease in surface tension is not sufficient to explain the differences: a small variation of temperature should have a higher effect than the presence of alcohol. Tap and demineralized waters have also very similar surface tensions. It means that other phenomena cause the differences in term of gas fraction and bubble size between the investigated systems. Some authors suggest that the differences are due to the reduction of the bubble-rise velocity (Kelkar et al., 1983). This explanation is reasonable in the case of homogeneous regime, where the bubbles rise vertically and the bubble velocity tends to the terminal velocity. Under the heterogeneous regime, the effect of macro-scale turbulence eclipses the other ones (Camarasa et al., 1999; Zahradník et al., 1995, 1997). 
As suggested by Krishna et al. (2000a), the effect on the gas fraction can be entirely explained with the delay of the transitional regime. The homogeneous regime is larger, causing a higher value of the gas hold-up of transition. The gas hold-up of transition in presence of alcohol can be estimated using the correlation of Reilly et al. (1994).

Lower coalescence causes a delay in the transition to the heterogeneous regime, as shown in Fig. 6 for a superficial gas velocity of $6 \mathrm{~cm} / \mathrm{s}$, as lower coalescence rate cause smaller bubbles, resulting in a higher gas hold-up into the column. In fact, bigger bubbles are subjected to a higher buoyancy force (in comparison with the drag force) and they are quicker to rise. When big bubbles are formed, they rise quickly in the center of the column, affecting the transition from the homogeneous regime to the transitional regime and to the heterogeneous regime. By using another measurement method, although not precise, Maximiano Raimundo (2015) measured the corresponding BSD and found that they are wide, but still unimodal for most cases, and bimodal with a predominant peak, for some others. The biggest bubbles of the BSD rise in the centre of the column.

Following Najafi et al. (2007), the main effect of alcohol is to modify the surface mobility of the bubbles. The bubble interface is mobile, in the case of pure systems. The liquid film between the approaching bubbles can drain out quickly. Alcohol forms a monolayer at the interface; this causes an additional resistance to the liquid film motion of the liquid film (Jamialahmadi and Miiller-Steinhagen, 1992). This additional resistance increases the time of liquid film drainage and it decreases the overall coalescence rate. Another important aspect is connected to the effect of the alcohol on the bubble 
zeta-potential, as studied by Takahashi (2005).

\subsection{Effect of the sparger}

The previous experiments were carried out using a sparger with 92 holes of $2 \mathrm{~mm}$ of diameter (Fig. 1b), ensuring a homogeneous gas distribution and a generation of rather small bubbles. The gas distribution is optimal, avoiding dead zones in the bubble column.

A new sparger is used to produce bigger bubbles and to induce bubble breakage in the lower part of the column. This sparger has 7 holes of 9 mm: 6 holes that form a hexagon and another hole in the center (Fig. 1c). The new sparger provides an injection that is completely different: the gas is injected heterogeneously in a small fraction of the column section. The gas is concentrated close to the holes. As the initial gas distribution is completely different, it is interesting to study if these differences have a local effect or if they influence the properties of the entire bubble column.

The axial gas fraction profiles obtain with the two spargers are compared in Fig. 8. In the case of heterogeneous regime, with demineralized water, more gas accumulates near the "heterogeneous" sparger in the column center. It is important to consider that in correspondence of the center of the column, where the data are collected, the new sparger has a hole (Fig. 1c). Close to the bottom of the column, using the "homogeneous" sparger, the radial profile of the gas fraction is almost flat (Fig. 9). Using the new sparger, the initial radial profile of the gas fraction changes: a strong accumulation is present close to the holes, while in the other radial positions the gas concentration is low. Above an axial position of $50 \mathrm{~cm}$, the gas fraction with the two sparger is approximately identical under the heterogeneous regime, as shown in Fig. 9. 


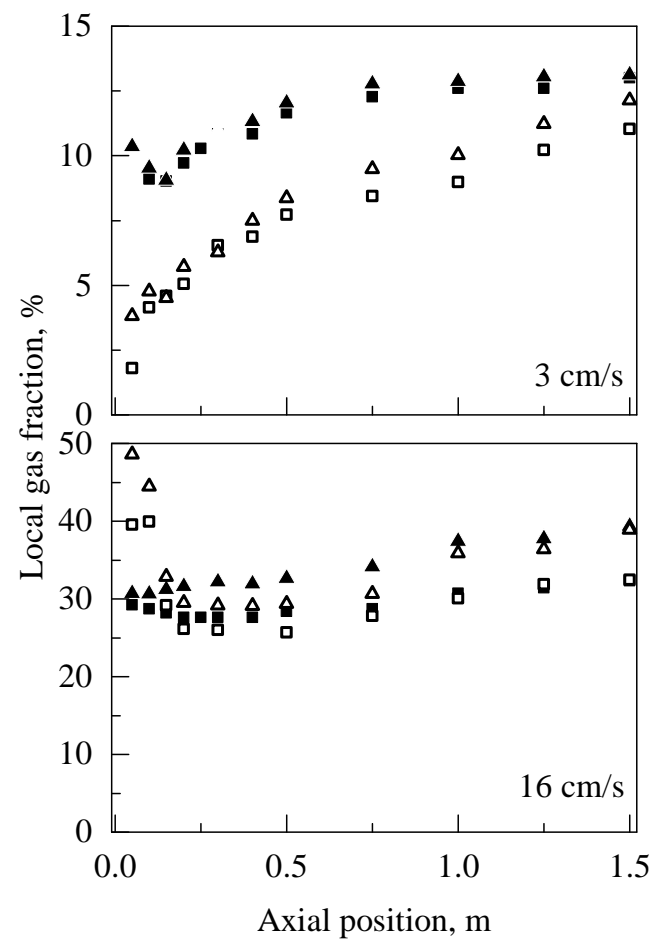

Figure 8: Axial gas fraction profile at the center of the column: sparger with 92 holes of $2 \mathrm{~mm}$ (with demineralized water (-) and ethanol $0.05 \%(\boldsymbol{\Lambda})$ ) vs sparger with 7 holes of 9 $\mathrm{mm}$ (with demineralized water $(\square)$ and ethanol $0.05 \%(\triangle)$ ) with two different superficial gas velocities: $3 \mathrm{~cm} / \mathrm{s}$ (top) and $16 \mathrm{~cm} / \mathrm{s}$ (bottom).

The effects of alcohol addition (ethanol 0.05\%) and the new sparger are coupled. In the case of heterogeneous regime, the sparger effect is the same with demineralized water and with alcohol addition: more gas accumulates near the sparger in the column center. At the same axial position, the gas fraction becomes lower using the new sparger. This effect becomes null after the first meter of the column; above this position, the gas fraction is not influenced by the sparger, both with demineralized water and with alcohol addition (Fig. 8). This aspect is reasonably consistent with the conclusion 


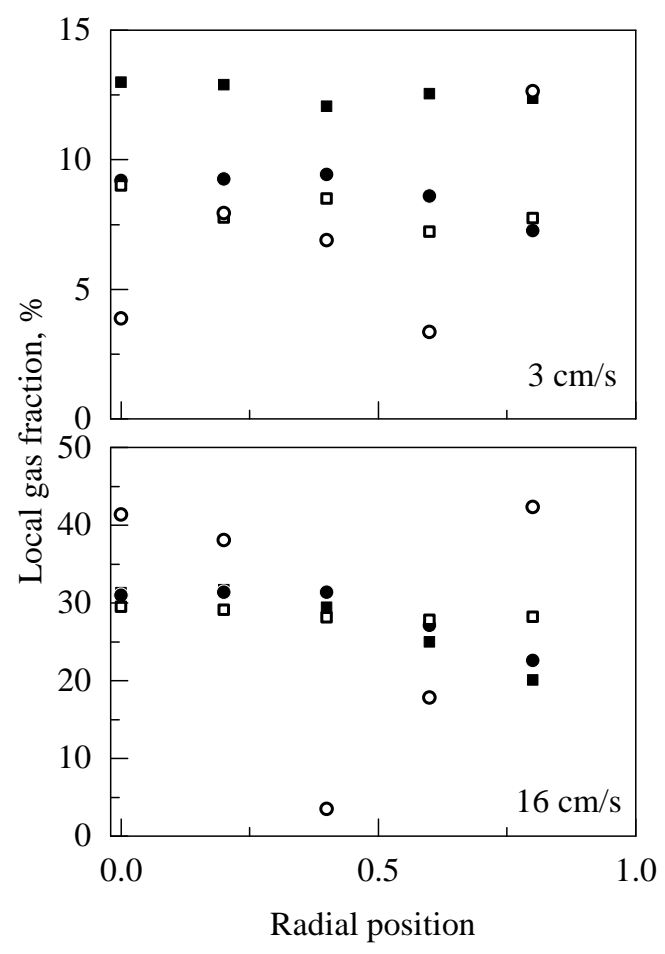

Figure 9: Radial gas fraction profile at different axial positions with demineralized water: sparger with 92 holes of $2 \mathrm{~mm}(\mathrm{H} / \mathrm{D}=2.5(\boldsymbol{\square})$ and $\mathrm{H} / \mathrm{D}=0.25(\square))$ vs sparger with 7 holes of $9 \mathrm{~mm}(\mathrm{H} / \mathrm{D}=2.5(\bullet)$ and $\mathrm{H} / \mathrm{D}=0.25(\mathrm{O}))$ with two different superficial gas velocities: 3 $\mathrm{cm} / \mathrm{s}$ (top) and $16 \mathrm{~cm} / \mathrm{s}$ (bottom).

of Polli et al. (2002), stating that the sparger effect is prevailing up to a distance of the order of magnitude of the column diameter.

Under the homogeneous regime, the sparger effect is important in each position of the column and this is the predominant effect for determining the gas fraction. Using the sparger with 7 holes of $9 \mathrm{~mm}$, in each position of the column center, the gas fraction is lower than using the "homogeneous" sparger. The alcohol addition has an effect that is less important than in the case of the heterogeneous regime (Fig. 8). 
The bubble size is strongly influenced by the sparger type, with the formation of very large bubbles (above $20 \mathrm{~mm}$ ) close to the bottom (Fig. 10) opposite to the holes (Fig. 11). The mean Sauter diameter decreases rapidly

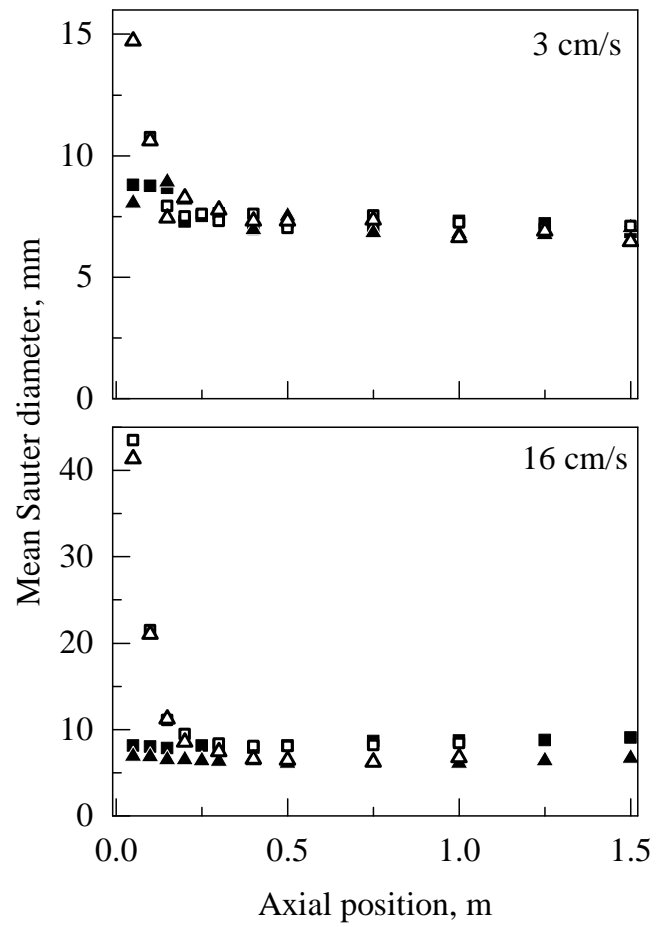

Figure 10: Axial mean Sauter diameter profile at the center of the column obtained with a sparger with 92 holes of $2 \mathrm{~mm}$ (with demineralized water (๘) and ethanol $0.05 \%(\mathbf{\Lambda})$ ) vs sparger with 7 holes of $9 \mathrm{~mm}$ (with demineralized water $(\square)$ and ethanol $0.05 \%(\triangle)$ ) with two different superficial gas velocities: $3 \mathrm{~cm} / \mathrm{s}$ (top) and $16 \mathrm{~cm} / \mathrm{s}$ (bottom).

along the axial position and after some centimeters the bubble size is almost identical to the case of "homogeneous" sparger (Fig. 11). In heterogeneous regime, the sparger has weak effect both on gas fraction and on Sauter diameter for $\mathrm{H} / \mathrm{D}>1$. It means that the initial bubble size does not influence the final bubble size in the case of heterogeneous regime. This effect is not 


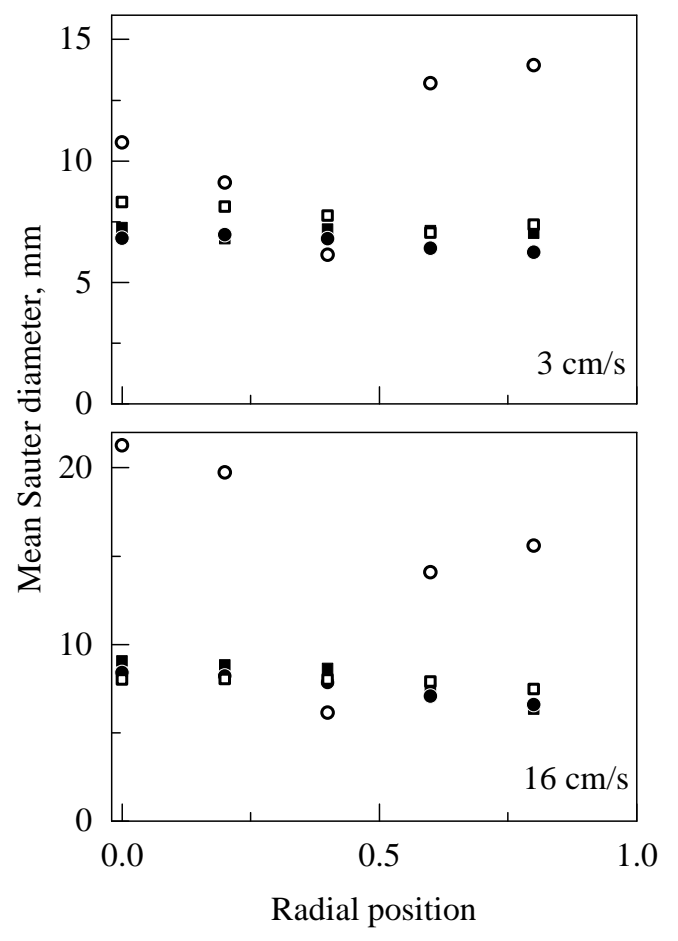

Figure 11: Radial mean Sauter diameter profile at different axial positions with demineralized water: sparger with 92 holes of $2 \mathrm{~mm}(\mathrm{H} / \mathrm{D}=2.5(\boldsymbol{\square})$ and $\mathrm{H} / \mathrm{D}=0.25(\square))$ vs sparger with 7 holes of $9 \mathrm{~mm}(\mathrm{H} / \mathrm{D}=2.5(\bullet)$ and $\mathrm{H} / \mathrm{D}=0.25(\mathrm{O}))$ with two different superficial gas velocities: $3 \mathrm{~cm} / \mathrm{s}$ (top) and $16 \mathrm{~cm} / \mathrm{s}$ (bottom).

negligible in the lowest part of the column. Close to the bottom, the radial profile of the mean Sauter diameter is flat using the "homogeneous" sparger, while it is irregular using the new sparger, as shown in Fig. 11. The irregularity of this profile is linked to the creation of random preferential paths of the bubbles. The effect of the sparger on the bubble size is only significant in the region near the bottom. Close to the bottom, the mean Sauter diameter is very high and bubble breakage is more important than bubble coalescence. Bubble breakage could be considered as the main phenomenon in this region, 
as in comparison bubble coalescence is negligible.

Under the heterogeneous regime, the final dimension of the bubbles is almost not influenced by the initial bubble size, while the alcohol presence is very important, as shown in Fig. 10. A noteworthy aspect is the rate of bubble size decrease along the axial position: the presence of alcohol almost does not influence the rate of bubble size decrease. The alcohol effect is important only when the bubble size reaches equilibrium. When the bubbles are huge, as in the lower part of the column with the 7-holes sparger, bubble breakage can be considered as much more important than bubble coalescence. Since bubble breakage could be considered as the main phenomenon in this region coalescence and break-up can be decoupled. The presence of alcohol can be used to infer coalescence and break-up rates. Experimental data show that the presence of the alcohol almost does not influence the rate with which bubbles coming out of the sparger reduce their size, but on the contrary it affects the final equilibrium size. From these experiments it can be inferred that the alcohol does not change significantly the break-up rate, but it reduces the coalescence rate. Contaminants influence the coalescence rate, while the influence on break-up is less important. This is consistent with the hypotheses previously expressed: the main alcohol effect is on the bubble surface mobility.

\section{Conclusions}

The main objective of this experimental work is to study the hydrodynamics and the bubble size in bubble columns under the heterogeneous regime, in order to highlight and study the coalescence and breakage phenomena. 
The use of different quality of water and the addition of small quantities of ethanol is useful to control coalescence. Bubbles in tap water are significantly smaller than in demineralized water. The presence of a small quantity of ethanol into demineralized water decreases the bubble size and increases the gas fraction in the heterogeneous regime. Adding ethanol, the transitional regime is delayed and higher gas fractions are observed under the heterogeneous regime. The alcohol presence modifies the surface mobility of the bubbles and it decreases bubble coalescence. Surface tension variations may not be the only valid explanation to these differences and further investigation concerning interface physical properties may be necessary to understand the way it inhibits coalescence.

A change of the sparger radically modifies the bubble size in the lowest part of the column. By using a perforated sparger with big holes, there is an accumulation of big bubbles close to the bottom, where bubble breakage is the predominant phenomenon. Coupling the effect of ethanol and the effect of the sparger, it is possible to observe that breakage is almost not influenced by the alcohol presence, confirming that its main effect is on bubble coalescence, through a change in the surface mobility.

Experimental results obtained during this study allow to identify some flow configurations where bubble generation and breakage are the only phenomena governing bubble size. Other configurations have also been found where coalescence inhibition by alcohol significantly modifies the bubble size. The use of both different spargers and alcohol is a good method to produce experimental data for breakage and coalescence modelling in heterogeneous bubble columns. Novel data presented in this study can already be used in 
this sense.

\section{Perspectives}

Adding some bubble size distributions (BSD) would be interesting, but unfortunately the main limit of the cross-correlation technique is the inability of calculating the BSD, as this method gives only the mean diameter. A great idea for future work could be to couple a classical optical probe for BSD measurement with a cross-correlation probe for mean bubble diameter measurement, in order to rescale the $\mathrm{BSD}$, being this latter not precisely measured in the heterogeneous regime.

The experimental data obtained in this work may be used for studying the coalescence and breakage phenomena. Coalescence models ought to take into account the impact of additives that drastically change the collective behavior of the gas-liquid dispersion without changing much the involved physical properties (i.e. surface tension). Their effect is related to the change in the mobility of the gas-liquid interface, that in turn affects the coalescence efficiency. Breakage is almost not influenced by the presence of ethanol, as stated by coupling ethanol and sparger effects.

Axial and radial profiles of mean bubble size under different operating conditions will be used to test different kernels and to tune the fitting parameters, as done for example for liquid-liquid dispersions (Gao et al., 2016; Li et al., 2017a,b). These kernels are generally based on considerations on the forces causing and opposing the coalescence and breakage events and on how these forces change when contaminants are present, that in turn affect the interface mobility. 
Velocity, gas fraction profiles and bubble size have a strong link with the closures for interfacial forces and turbulence. The PBM is solved within CFD correctly predicting velocity and gas fraction profiles is of paramount importance. The PBM depends on bubble breakup and coalescence and on the hydrodynamics. At the same time, these profiles depend on the bubble size: it is necessary to have a correct PBM for simulating the hydrodynamics. Concluding, hydrodynamics and PBM ought to be coupled for obtaining a good modeling of these systems.

\section{Acknowledgements}

The authors want to thank Grace Bobe Mboyo and Vincenzo Cappello for their contribution to these experiments. Computational resources were provided by HPC@POLITO, a project of Academic Computing within the Department of Control and Computer Engineering at the Politecnico di Torino (http://www.hpc.polito.it).

\section{References}

Camarasa, E., Vial, C., Poncin, S., Wild, G., Midoux, N. and Bouillard, J. (1999), 'Influence of coalescence behaviour of the liquid and of gas sparging on hydrodynamics and bubble characteristics in a bubble column.', Chemical Engineering and Processing 38, 329-344.

Chaumat, H., Billet, A. and Delmas, H. (2007), 'Hydrodynamics and mass transfer in bubble column: Influence of liquid phase surface tension.', Chemical Engineering Science 62, 7378-7390. 
Clift, R., Grace, J. R. and Weber, M. E. (1978), Bubbles, Drops, and Particles., Academic Press, New York, New York.

Dargar, P. and Macchi, A. (2006), 'Effect of surface-active agents on the phase holdups of three-phase fluidized beds.', Chemical Engineering and Processing 45, 764-772.

Deckwer, W. (1992), Bubble Column Reactors., Wiley, Chichester, New York.

Forret, A. (2003), Hydrodynamics scale-up of slurry bubble columns., PhD thesis, Universit Claude Bernard Lyon 1 - IFPEN, Lyon.

Gao, Z., Li, D., Buffo, A., Podgrska, W. and Marchisio, D. L. (2016), 'Simulation of droplet breakage in turbulent liquid-liquid dispersions with CFD-PBM: Comparison of breakage kernels', Chemical Engineering Science 142, 277-288.

Guo, K., Wang, T., Liu, Y. and Wang, J. (2017), 'CFD-PBM simulations of a bubble column with different liquid properties', Chemical Engineering Journal 329(Supplement C), 116-127. XXII International conference on Chemical Reactors CHEMREACTOR-22.

Jakobsen, H. A., Lindborg, H. and Dorao, C. A. (2005), 'Modeling of bubble column reactors: Progress and limitations', Industrial \&3 Engineering Chemistry Research 44(14), 5107-5151.

Jamialahmadi, M. and Miiller-Steinhagen, H. (1992), 'Inhibition of bubble coalescence by solutes in air/water dispersions.', Chemical Engineering Journal 50, 47-56. 
Keitel, G. and Onken, U. (1982), 'Inhibition of bubble coalescence by solutes in air/water dispersions.', Chemical Engineering Science 37, 1635-1638.

Kelkar, B. G., Godbole, S. P., Honath, M. F. and Shah, Y. T. (1983), 'Effect of addition of alcohols on gas holdup and backmixing in bubble columns.', AIChE Journal 29, 361-369.

Krishna, R. (2000), 'A scale-up strategy for a commercial scale bubble column slurry reactor for Fischer-Tropsch synthesis.', Oil \& Gas Science and Technology 55(4), 359-393.

Krishna, R., Dreher, A. J. and Urseanu, M. (2000a), 'Influence of alcohol addition on gas hold-up in bubble columns: Development of a scale up model.', International Communications in Heat and Mass Transfer 27(4), 465-472.

Krishna, R., Urseanu, M., Van Baten, J. and Ellenberger, J. (2000b), 'Liquid phase dispersion in bubble columns operating in the churn-turbulent flow regime', Chemical Engineering Journal 78(1), 43 - 51.

Li, D., Buffo, A., Podgrska, W., Marchisio, D. L. and Gao, Z. (2017a), 'Investigation of droplet breakup in liquidliquid dispersions by CFDPBM simulations: The influence of the surfactant type', Chinese Journal of Chemical Engineering 25(10), 1369-1380.

Li, D., Gao, Z., Buffo, A., Podgorska, W. and Marchisio, D. L. (2017b), 'Droplet breakage and coalescence in liquidliquid dispersions: Comparison of different kernels with EQMOM and QMOM', AIChE Journal 63(6), 2293-2311. 
Liao, Y. and Lucas, D. (2009), 'A literature review of theoretical models for drop and bubble breakup in turbulent dispersions.', Chemical Engineering Science 64, 3389-3406.

Liao, Y. and Lucas, D. (2010), 'A literature review on mechanisms and models for the coalescence process of fluid particles.', Chemical Engineering Science 65, 2851-2864.

Lindland, K. P. and Terjesen, S. G. (1965), 'The effect of a surface-active agent on mass transfer in falling drop extraction.', Chemical Engineering Science 5(1), 1-12.

Marchisio, D. L. and Fox, R. O. (2013), Computational Models for Polydisperse Particulate and Multiphase Systems., Cambridge University Press, Cambridge, UK.

Maximiano Raimundo, P. (2015), Analysis and modelization of local hydrodynamics in bubble columns., PhD thesis, Universit Grenoble Alpes.

Maximiano Raimundo, P., Cartellier, A., Beneventi, D., Forret, A. and Augier, F. (2016), 'A new technique for in-situ measurements of bubble characteristics in bubble columns operated in the heterogeneous regime', Chemical Engineering Science 155, $504-523$.

McClure, D. D., Kavanagh, J. M., Fletcher, D. F. and Barton, G. W. (2013), 'Development of a CFD model of bubble column bioreactors: Part one a detailed experimental study', Chemical Engineering $\& 3$ Technology 36(12), 2065-2070. 
McClure, D. D., Kavanagh, J. M., Fletcher, D. F. and Barton, G. W. (2017), 'Experimental investigation into the drag volume fraction correction term for gas-liquid bubbly flows', Chemical Engineering Science 170, 91-97. 13th International Conference on Gas-Liquid and Gas-Liquid-Solid Reactor Engineering.

McClure, D. D., Norris, H., Kavanagh, J. M., Fletcher, D. F. and Barton, G. W. (2015), 'Towards a CFD model of bubble columns containing significant surfactant levels.', Chemical Engineering Science 127, 189-201.

McClure, D. D., Wang, C., Kavanagh, J. M., Fletcher, D. F. and Barton, G. W. (2016), 'Experimental investigation into the impact of sparger design on bubble columns at high superficial velocities.', Chemical Engineering Research and Design 106, 205-213.

Miyauchi, T. and Shyu, C.-N. (1970), 'Flow of fluid in gas-bubble columns', Chemical engineering 34(9), 958-964.

Najafi, A. S., Drelich, J., Yeung, A., Xu, Z. and Masliyah, J. (2007), 'A novel method of measuring electrophoretic mobility of gas bubbles.', Journal of Colloid and Interface Science 308(2), 344-350.

Polli, M., Stanislao, M. D., Bagatin, R., Bakr, E. A. and Masi, M. (2002), 'Bubble size distribution in the sparger region of bubble columns', Chemical Engineering Science 57(1), 197-205.

Ranade, V. (2002), Computational Flow Modeling for Chemical Reactor Engineering., Academic Press, San Diego, California, USA. 
Raymond, D. R. and Zieminski, S. A. (1971), 'Mass transfer and drag coefficients of bubbles rising in dilute aqueous solutions.', AIChE Journal 17(1), 57-65.

Reilly, I. G., Scott, D. S., De Bruijn, T. J. W. and MacIntyre, D. (1994), 'The role of gas phase momentum in determining gas hold-up and hydrodynamic flow regimes in bubble column operations.', The Canadian Journal of Chemical Engineering 72, 3-12.

Sasaki, S., Uchida, K., Hayashi, K. and Tomiyama, A. (2017), 'Effects of column diameter and liquid height on gas holdup in air-water bubble columns.', Experimental Thermal and Fluid Science 82, 359-366.

Schweitzer, J.-M. (2001), 'Local gas hold-up measurements in fluidized bed and slurry bubble column.', Chemical Engineering Science 56(3), 11031110.

Takahashi, M. (2005), ' $\zeta$ potential of microbubbles in aqueous solutions: electrical properties of the gaswater interface.', The Canadian Journal of Chemical Engineering 109(46), 21858-21864.

Tisnadjaja, D., Gutierrez, N. A. and Maddox, I. S. (1996), 'Citric acid production in a bubble-column reactor using cells of the yeast Candida guilliermondii immobilized by adsorption onto sawdust', Enzyme and Microbial Technology 19(5), 343-347.

Vial, C., Bendjaballah-Lalaoui, N., Poncin, S., Wild, G. and Midoux, N. (2003), 'Comparison, combination and validation of measuring techniques 
for local flow and turbulence analysis in bubble columns and airlift reactors.', The Canadian Journal of Chemical Engineering 81(3-4), 749-755.

Wellek, R. M., Agrawal, A. K. and Skelland, A. H. P. (1966), 'Shape of liquid drops moving in liquid media', AIChE Journal 12(5), 854-862.

Xiao, Q., Wang, J., Yang, N. and Li, J. (2017), 'Simulation of the multiphase flow in bubble columns with stability-constrained multi-fluid CFD models', Chemical Engineering Journal 329(Supplement C), 88-99. XXII International conference on Chemical Reactors CHEMREACTOR-22.

Xue, J. (2004), Bubble velocity, size and interfacial area measurements in bubble columns., PhD thesis, Sever Institute of Washington University.

Zahradník, J., Fialov, M., Kaštánek, F., Green, K. and Thomas, N. (1995), 'The effect of electrolytes on bubble coalescence and gas hold-up in bubble column reactors.', Chemical Engineering Research and Design 73, 341-346.

Zahradník, J., Fialov, M., Røužička, M., Drahoš, J., Kaštánek, F. and Thomas, N. (1997), 'Duality of the gas-liquid flow regimes in bubble column reactors', Chemical Engineering Science 52(21), 3811-3826. 